LA W RENCE LIVERM ORE NATIONAL LABORATORY
A Time Dependent ODE Conversion Of The Detonation Shock Dynamics

Evolution Equation

J. Yao

August 6, 2013 
This document was prepared as an account of work sponsored by an agency of the United States government. Neither the United States government nor Lawrence Livermore National Security, LLC, nor any of their employees makes any warranty, expressed or implied, or assumes any legal liability or responsibility for the accuracy, completeness, or usefulness of any information, apparatus, product, or process disclosed, or represents that its use would not infringe privately owned rights. Reference herein to any specific commercial product, process, or service by trade name, trademark, manufacturer, or otherwise does not necessarily constitute or imply its endorsement, recommendation, or favoring by the United States government or Lawrence Livermore National Security, LLC. The views and opinions of authors expressed herein do not necessarily state or reflect those of the United States government or Lawrence Livermore National Security, LLC, and shall not be used for advertising or product endorsement purposes.

This work performed under the auspices of the U.S. Department of Energy by Lawrence Livermore National Laboratory under Contract DE-AC52-07NA27344. 


\title{
A Time Dependent ODE Conversion Of The Detonation Shock Dynamics Evolution Equation
}

\author{
Yao, Jin \\ Lawrence Livermore National Laboratory, California, USA
}

\begin{abstract}
The equation of motion for a detonation front propagation under a $D_{n}-\kappa$ law is converted to a set of ordinary differential equations in the front normal coordinate for a third order spatial accuracy. Which allows a time integration of the system with an ODE integrator for a high accuracy.
\end{abstract}

\section{INTRODUCTION}

The propagation of a detonation wave in a homogeneous HE material can be described with the theory of detonation shock dynamics (DSD). As the DSD theory can accurately predict the position of a detonation front with much less cost compared to DNS, especially in three-dimensions, employing DSD wherever possible is desired.

To the leading order approximation of the DSD theory, the detonation front velocity in the normal direction is a function of the front curvature, or a $D_{n}-\kappa$ law $D_{n}=D_{n}(\kappa)$, where $D_{n}$ is the detonation velocity and $\kappa$ is the front curvature. The motion of the front is described by a partial differential equation

$$
\partial \phi / \partial t+D_{n}|\nabla \phi|=0
$$

where $\phi(\vec{r}, t)=0$ defines the position of the front in space and time.

To track the front with a $D_{n}-\kappa$ law, a third order of spatial accuracy is required for a proper evaluation of the curvature. In the local front normal coordinate (the shock-attached frame), the detonation front can be expressed with a local quadratic expansion of spatial coordinates. Furthermore the equation of motion is converted to a set of ordinary differential equations of the time dependent coefficients that define the expansion. Then the time integration of the equation of motion can be performed with an ODE integrator of high accuracy, such as a Runge-Kutta method. 


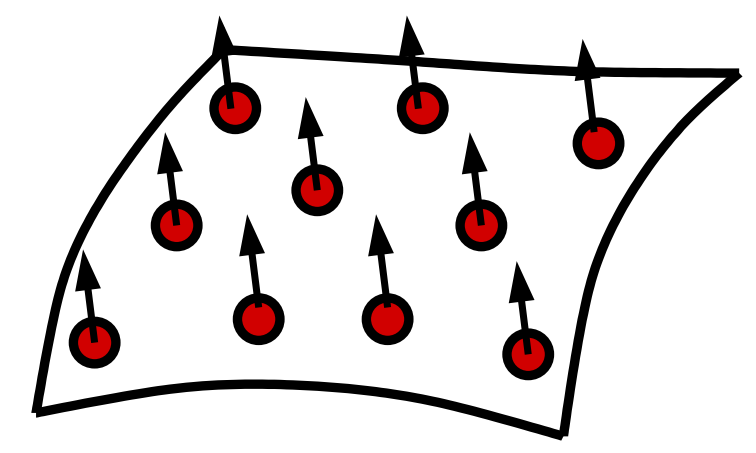

Figure 1: A propagating detonation front represented by a set of particles moving in the normal direction.

A detonation front can be represented with marked particles, the motion of each particle in the normal direction has to be solved (fig. 1). In the case of determining the signed-distances for a point set to a propagating detonation front, the relative motion between the front and a given point also needs to be solved as well. In both cases, the ODE system derived in this paper would help to obtain an accurate time integration. The trade off is a $3^{\text {rd }}$ order error in space. Such an accuracy is required for evaluation of curvature and is rather acceptable in almost every numerical simulation.

In this paper the derivation of the time dependent ODE system from the PDE form of the evolution equation of detonation shock dynamics is given. The accuracy with various time integration schemes are examined numerically against an exact integral of the ODE system for an linear $D_{n}-\kappa$ relation.

\section{IN THE FRONT NORMAL COORDINATE}

The normal vector at a point $\mathbf{P}$ on the front $\phi(\vec{r}, t)=0$ is defined by

$$
\vec{n}=\frac{\vec{\nabla} \phi}{|\nabla \phi|} .
$$

In three-dimensions, a local Cartesian coordinate system $(\xi, \eta, n)$ can be defined by setting the origin at $\mathbf{P}$ with the normal at $\mathbf{P}$ be the $n$-axis, $\xi$ and $\eta$ are the 


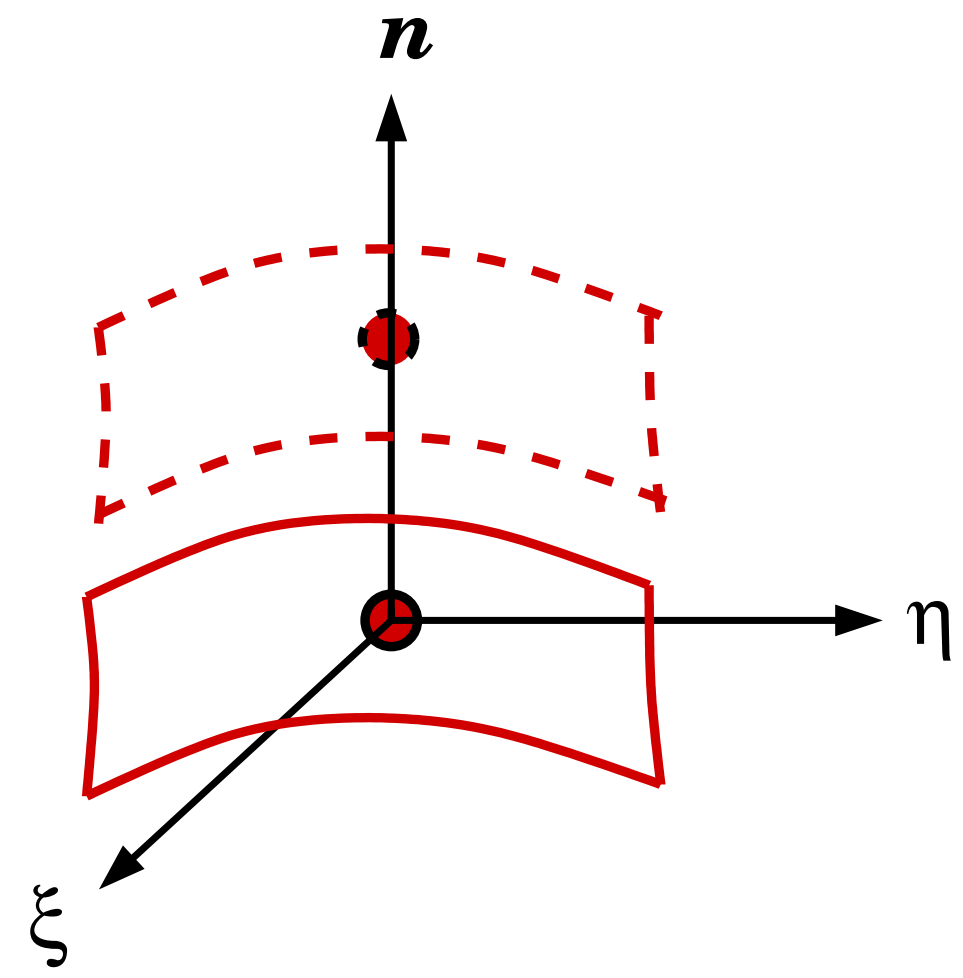

Figure 2: A detonation front propagates for a time step, in the local surface normal coordinate. The origin is set at a particle.

tangential unit vectors (fig. 2). In this front normal coordinate, a surface can be generally expressed by $n=h(\xi, \eta)$. We assume the surface that represents a detonation front is sufficiently smooth to well define the curvature. Such a surface may have an expansion in the form of

$$
n=\frac{a}{2} \xi^{2}+b \xi \eta+\frac{c}{2} \eta^{2}+e \xi+f \eta+g+\text { h.o.t... }
$$

to the third order of spatial accuracy the front, Then the front position can be expressed by $\phi=n-f(\xi, \eta)$ at any given time, with

$$
h(\xi, \eta)=\frac{a}{2} \xi^{2}+b \xi \eta+\frac{c}{2} \eta^{2}+e \xi+f \eta+g .
$$

The coefficients $a, b, c, e, f$, and $g$ are considered functions of time, to carry the change of geometry of the detonation front. 
Some special properties of such an expansion associated with the surface normal coordinate can be derived. Let us consider the moment $t=t_{0}$ (without loss of generality $t_{0}$ can be set to 0$)$. First of all because the origin is set at $(\xi, \eta)=(0,0)$, one must have $g(0)=0$.

We compute the normal vector by $\hat{e}_{n}=\vec{\nabla} \phi /|\nabla \phi|$, and with our choice of $\phi$ we have $\vec{\nabla} \phi=\hat{e}_{n}-(a \xi+b \eta+e) \hat{e}_{\xi}-(c \eta+b \xi+f) \hat{e}_{\eta}$. At the origin $(\xi, \eta)=(0,0)$ there must be only the $\hat{e}_{n}$ component therefore we also have $e(0)=0$ and $f(0)=0$.

The system can be further simplified by rotating the front normal frame around the $n$-axis to get rid of the cross term at $t=0$ thus we can set $b(0)=0$ also.

\section{THE EVOLUATION EQUATION CONVERTED TO AN ODE SYSTEM}

Plugging our choice of $\phi$ into the equation of motion that $\partial \phi / \partial t+D_{n}|\nabla \phi|=0$, one arrives at

$$
\frac{\dot{a}}{2} \xi^{2}+\dot{b} \xi \eta+\frac{\dot{c}}{2} \eta^{2}+\dot{e} \xi+\dot{f} \eta+\dot{g}=D_{n} \sqrt{A \xi^{2}+B \xi \eta+C \eta^{2}+E \xi+F \eta+G},
$$

where

$A=a^{2}+b^{2}, B=2 b(a+c), C=c^{2}+b^{2}, E=2(a e+b f), F=2(c f+b e), G=1+e^{2}+f^{2}$.

We are interested in the motion of a piece of front that contains the origin with the time integration of the system over a time step, thus we can expand the equation for small $\xi$ and $\eta$ to obtain

$$
\frac{\dot{a}}{2} \xi^{2}+\dot{b} \xi \eta+\frac{\dot{c}}{2} \eta^{2}+\dot{e} \xi+\dot{f} \eta+\dot{g}=D_{n}\left(\tilde{A} \xi^{2}+\tilde{B} \xi \eta+\tilde{C} \eta^{2}+\tilde{E} \xi+\tilde{F} \eta+\tilde{G}\right)+\text { h.o.t. }
$$

where

$$
\begin{gathered}
\tilde{A}=\frac{A}{2 \sigma}-\frac{E^{2}}{8 \sigma^{3}}, \tilde{B}=\frac{B}{2 \sigma}-\frac{E F}{4 \sigma^{3}}, \tilde{C}=\frac{C}{2 \sigma}-\frac{F^{2}}{8 \sigma^{3}}, \\
\tilde{E}=\frac{E}{2 \sigma}, \tilde{F}=\frac{F}{2 \sigma}, \tilde{G}=\sigma,
\end{gathered}
$$

$\sigma=\tilde{G}=\sqrt{1+e^{2}+f^{2}}$, and h.o.t. stands for higher order terms that are ignored in this analysis. 
Because $\xi$ and $\eta$ are arbitrary, one must have

$$
\begin{aligned}
& \frac{\dot{a}}{2}=D_{n} \tilde{A}, \dot{b}=D_{n} \tilde{B}, \frac{\dot{c}}{2}=D_{n} \tilde{C}, \\
& \dot{e}=D_{n} \tilde{E}, \dot{f}=D_{n} \tilde{F}, \dot{G}=D_{n} \tilde{G} .
\end{aligned}
$$

However, because $e(0)=0$ and $f(0)=0$, from $\dot{e}=D_{n} \tilde{E}$ and $\dot{f}=D_{n} \tilde{F}$ and the expressions of $E$ and $F$, we see that $e=0$ and $f=0$ satisfy both their initial conditions and the ordinary differential equations that governs their time development. A similar observation concludes that $b=0$ as well. This should not be a surprise because the front profile at $t=0$ is symmetric to the $n$-axis in which the front propagates and the equation for time development is also symmetric.

With $e=0, f=0$ and $b=0$, one has $\sigma=1, \tilde{A}=a^{2}, \tilde{C}=c^{2}$ and $\tilde{G}=1$. The ODE system can further be simplified to

$$
\dot{a}=a^{2} D_{n}(\kappa), \dot{c}=c^{2} D_{n}(\kappa), \dot{g}=D_{n}(\kappa) .
$$

The front curvature $\kappa$ is computed with $\kappa=\vec{\nabla} \cdot \hat{n}$. Because $\hat{n}=\vec{\nabla} \phi /|\nabla \phi|$ and

$$
\vec{\nabla} \phi=\vec{\nabla}\left(n-\frac{a}{2} \xi^{2}-\frac{c}{2} \eta^{2}-g\right)
$$

therefore

$$
\hat{n}=\frac{\hat{e}_{n}-a \xi \hat{e}_{\xi}-c \hat{\eta} e_{\eta}}{\sqrt{1+a^{2} \xi^{2}+c^{2} \eta^{2}}}
$$

and

$$
\kappa=\vec{\nabla} \cdot \hat{n}=-\frac{a+c+a c\left(a \xi^{2}+c \eta^{2}\right)}{\left(1+a^{2} \xi^{2}+c^{2} \eta^{2}\right)^{3 / 2}} .
$$

In the case that the front is represented by a distribution of marked particles, we are interested in the motion of a particle in the normal direction. Setting the origin of the local front normal frame to the position of the particle provides $\xi=0$ and $\eta=0$ and we have the curvature of the front on the path of the particle that

$$
\kappa=-(a+c),
$$

and only the first and the third equations in (eq. 2) are coupled together. 


\section{AN ANALYTICAL INTEGRAL WITH A SIM- PLE $D_{n}-\kappa$ RELATION}

The coupled governing equations (eq. 2) clearly tell that

$$
\frac{\dot{a}}{a^{2}}=\frac{\dot{c}}{c^{2}}=D_{n}(\kappa) .
$$

With the substitution of variables that $u=-1 / a$ and $v=-1 / c$ into the equations, the the ODE system is further reduced to

$$
\dot{u}=D_{n}(\kappa), \dot{v}=D_{n}(\kappa), \quad \text { and } \quad \dot{g}=D_{n}(\kappa) .
$$

One obtains immediately a first integral that

$$
v=u+2 \beta,
$$

where $\beta$ is a time independent constant which makes the above equation true at $t=0$, thus

$$
\beta=\frac{v(0)-u(0)}{2} .
$$

An analytical solution exists for a linear form of the $D_{n}-\kappa$ relation that

$$
D_{n}=D_{C J}(1-\alpha \kappa),
$$

where $D_{C J}$ is the CJ velocity of a detonation and can be taken to one with a time scaling. For simplicity we assume $D_{n}=1-\alpha \kappa, \alpha$ is the slope of the $D_{n}-\kappa$ curve, the total curvature is computed as

$$
1 / u+1 / v
$$

We can eliminate $v$ from the governing equation to obtain

$$
\dot{u}=D_{n}(\kappa)=1-\alpha\left(\frac{1}{u}+\frac{1}{2 \beta+u}\right) .
$$

Thus

$$
d t=\frac{d u}{1-\alpha(1 / u+1 /(2 \beta+u))},
$$


then one obtains an explicit integral that

$$
t-\gamma=u+\frac{2 \alpha^{2}}{\sqrt{\alpha^{2}+\beta^{2}}} \operatorname{ArcTanh}\left(\frac{\alpha+\beta+u}{\sqrt{\alpha^{2}+\beta^{2}}}\right)-\alpha \log \left|(u+\alpha+\beta)^{2}-\left(\alpha^{2}+\beta^{2}\right)\right|,
$$

where $\gamma$ is a constant, which would make $t=0$ when $u=u(0)$ and $v=v(0)$.

The time integration of $\dot{g}=D_{n}(\kappa)$ now can be related to $u$ with another first integral that

$$
\dot{g}-\dot{u}=0,
$$

because the particle was at the origin at $t=0$, therefore $g(0)=0$ and

$$
g=u-u(0) .
$$

This is easy to understand considering that $g$ is the distance traveled by a particle on the normal-axis and $u=-1 / a$ is equivalent to the radius of curvature in the case of a spherical wave.

An analytical integral shall be helpful in examining the accuracy of a numerical time integration scheme.

\section{NUMERICAL TEST WITH A LINEAR $D_{n}-\kappa$}

To obtain the position of a particle moving in the surface normal direction with the definition

$$
d r / d t=D_{n}(\kappa)
$$

without utilizing the geometry of the front, an explicit way is to use a forward Euler method for a first order of accuracy in time. A predictor-corrector method may be implemented for a second order accuracy with the curvature at the next time step becomes available after all the particles are updated (with the explicit Euler method).

The proposed method solves the evolution equation of the front (a PDE) locally by converting the governing equation to a set of ODEs with a third order of spatial accuracy. The time integration can be as accurate as arbitrarily required with an existing ODE integrator.

To demonstrate the better accuracy with solving the ODE system derived previously, compared to a common numerical scheme, we employ a linear $D_{n}-\kappa$ relation 
$D_{n}=1-\alpha \kappa$ and integrate it for a time step $\Delta t$. The result is checked against the analytical integral obtained in the section above.

For simplicity we also take that $u(0)=v(0)$ therefore $\beta=0$ and we have an axi-symmetry case here. The analytical integral (eq. 9) then is reduced to

$$
t=\gamma+u+2 \alpha \log |u-2 \alpha|
$$

We pick $\alpha=0.1, u(0)=v(0)=1$ for the calculation in this section. The distance traveled by a given particle on the front initially described with the above parameters is computed, with both the forward Euler method, the Trapezoid method, and the proposed method with a $4^{\text {th }}$ order Runga-Kutta method, for a variable size of $d t$. The results obtained are compared with the analytical solution for an evaluation of accuracy.

The comparison between results from various time integration methods are shown in the table below

\begin{tabular}{|c|c|c|c|c|c|}
\hline$\Delta t$ & $E_{\text {Euler }}$ & $E_{\text {Trapezoid }}$ & $E_{R K 4(1)}$ & $E_{R K 4(2)}$ & distance \\
\hline 0.1 & 0.000764 & 0.000017 & 0.000000 & 0.000000 & 0.080764 \\
\hline 0.2 & 0.002927 & 0.000125 & 0.000000 & 0.000000 & 0.162927 \\
\hline 0.4 & 0.010078 & 0.000846 & 0.000005 & 0.000000 & 0.330788 \\
\hline 0.8 & 0.037325 & 0.005020 & 0.000082 & 0.000006 & 0.677325 \\
\hline 1.6 & 0.117873 & 0.024599 & 0.001014 & 0.000097 & 1.397873 \\
\hline
\end{tabular}

Table 1: The comparison of accuracy in time integration with a linear $D_{n}-\kappa$ relation $D_{n}=1-0.1 \kappa$ for an axi-symmetric detonation propagation.

the letter $E$ stands for absolute numerical error against the exact solution. $E_{E u l e r}$, $E_{\text {Trapezoid }}, E_{R K 4(1)}$, and $E_{R K 4(2)}$ stand for the absolute errors with the distance traveled by a marked particle in a time step compared to the exact solution (eq. $10)$ of the ODE system reduced from the DSD evolution equation with a $3^{\text {rd }}$ order spatial accuracy. $R K 4(1)$ for a one step $4^{\text {th }}$ order Runga-Kutta integration, $R K 4(2)$ for a two step one. distance is the distance traveled by a marked particle on the detonation front during the time step.

Without introducing the ODE system described above, the time integration of the DSD evolution equation with a marked particle representation of the detonation front would be uneasy to implement for the Trapezoid method because curvature cannot be easily updated. A Runga-Kutta method with multiple steps is nearly 
impossible to implement for the same reason. Thus with a trade off on spatial accuracy to $3^{\text {rd }}$ order (which is sufficient to adequately handle curvature), one is able to obtain arbitrarily accurate time integration with the method described in this paper by solving a simple ODE system.

\section{CONCLUSION}

The evolution equation for a detonation front described by detonation shock dynamics is locally converted to a set of simple ODE system with a $3^{\text {rd }}$ order spatial accuracy. The motion of a point on the detonation front at the next time step then can be accurately integrated in time with an ODE integrator of choice. For a detonation represented by a set of marked particles, such an conversion allows an easy implementation of accurate time integration for each particle. If we are not seeking a spatial accuracy higher than $3^{\text {rd }}$ order, the method proposed in this paper would provide an ideal solution with time advancement for tracking the propagation of a detonation using a point representation of the front.

\section{REFERENCES}

[1]. Level-Set Techniques Applied to Modeling Detonation Shock Dynamics, T. Aslam, J. Bdzil, and D. S. Stewart, Journal of Computational Physics, 126, 390409, 1996.

[2]. On the Dynamics of Multi-dimensional Detonation, J. Yao, and D. S. Stewart, Journal of Fluid Mechanics, 309, 225-275, 1996.

[3]. Level Set Methods and Fast Marching Methods, J.A. Sethian, Cambridge University Press, 1999.

[4]. A Geometrical DSD Lighting Algorithm on Arbitrary Unstructured Meshes, J. Yao, UCRL-JRNL-213932, Lawrence Livermore National Laboratory, 2005.

[5]. Test Problems for DSD2D, John B. Bdzil, Rudolph J. Henninger, and John, W. Walter, LA-14277, 2006.

[6]. The Dynamics of Detonation in Explosive Systems, John B. Bdzil, and D. Scott Stewart, Annual Review of Fluid Mechanics, Vol. 39:263-292, 2007.

[7]. A General Dead-Zone Predicted With The Detonation Shock Dynamics, J. Yao, LLNL-TR-640104, 2013 